\title{
Combinatorial estimates by the switching method
}

\author{
Mahdieh Hasheminezhad and Brendan D. McKay
}

\begin{abstract}
The method of switchings is a standard tool for enumerative and probabilistic applications in combinatorics. In its simplest form, it analyses a relation between two sets to estimate the ratio of their sizes. In a more complicated setting, there is a family of sets connected by some relations. By bounding properties of the relations, bounds can be inferred on the relative sizes of the sets. In this paper we extend the treatment of Fack and McKay (2007) to allow the graph of sets and relations to be an arbitrary directed graph. A special case that frequently occurs in bounding tails of distributions is analysed in detail.
\end{abstract}

\section{Introduction}

The simplest example of the method of switchings involves two disjoint finite sets $A, B$, and a relation $R \subseteq A \times B$. If $d_{A}$ is the average number of elements of $B$ that are related to a uniformly chosen random element of $A$, and $d_{B}$ is the average number of elements of $A$ that are related to a uniformly chosen random element of $B$, then $d_{A}|A|=|R|=d_{B}|B|$. Thus, estimates of the relative values of $d_{A}$ and $d_{B}$ provide estimates of the relative sizes of $A$ and $B$.

Frequently the relation comes from some operation, called a switching for historical reasons, that takes an object in $A$ and modifies it to make an object in $B$. In this view, $d_{A}$ is the average number of switchings that can be applied to an object of $A$, and $d_{B}$ is the average number of switchings that can make an object of $B$.

In more complex situations, we might have a large number of disjoint sets and a switching operation that maps objects in the sets to objects in the same or different sets. Our aim then is to infer bounds on the relative sizes of the sets by analysing the switchings. We can model the overall structure by means of a directed graph that we will call the structure graph $G=(V, E)$. Each $v \in V$ is associated with a finite set $C(v)$, these sets being disjoint. Moreover, whenever there are objects $Q \in C(v), R \in C(w)$ such that $Q$ can be taken onto $R$ by a switching, there is a directed edge $(v, w)$ in $E$.

2010 Mathematics Subject Classification. 05A16; 05A20, 60C05.

Current address: Department of Computer Science, Faculty of Mathematics, Yazd University, Yazd, 89195-741, Iran.

Research supported by the Australian Research Council.

(c) 0000 (copyright holder) 
There are many examples in the literature where classes of combinatorial objects are approximately enumerated by this technique in the case that $G$ is a directed path. A few examples are $[\mathbf{1}, \mathbf{3}, \mathbf{6}, \mathbf{7}, \mathbf{8 ,}$, 10, 11]. Fack and McKay [2] gave a more general analysis, allowing $G$ to be an arbitrary acyclic directed graph, plus optional loops. An example application is given in [4]. In this case precise estimates are hard to obtain but the technique provides good tail bounds in many cases.

The advantage of the method is its very wide applicability. For bounding tails of distributions, it is an additional technique to add to the existing toolkit that includes martingales and other methods. See [5] for a survey.

In this paper, we complete the analysis of Fack and McKay by allowing $G=$ $(V, E)$ to be an arbitrary finite directed graph. Loops are allowed, but multiple edges are not (they have no evident use). We begin with a formal statement of the problem. Define $C(V)=\bigcup_{v \in V} C(v)$. Let $\mathcal{S}$ be a multiset of ordered pairs of elements of $C(V)$ such that $(Q, R) \in \mathcal{S}$ for $Q \in C(v), R \in C(w)$, then $(v, w) \in E$. Let $X, Y \subseteq V$. For each $v \in V$, define $N(v)=|C(v)|$, and for $W \subseteq V$, define $N(W)=\sum_{w \in W} N(w)$. For $(v, w) \in E$, consider the multiset cardinality

$$
s^{\prime}(v w)=|\{(Q, R) \in \mathcal{S}: Q \in C(v), R \in C(w)\}|
$$

For a vertex $v \in V$, let $G^{-}(v)$ and $G^{+}(v)$ be the set of (directed) edges entering and leaving $v$, respectively. Suppose that on average an object in $C(v)$ can be subject to at least $a(v)$ switchings and can be produced by at most $b(v)$ switchings. Then we have the following optimisation problem.

Problem A. Suppose that $a, b: V \rightarrow \mathbb{R}$ are positive functions. Maximise $N(Y) / N(X)$ subject to

$$
\begin{aligned}
N(v) & \geq 0 & & \text { for all } v \in V \\
s^{\prime}(v w) & \geq 0 & & \text { for all } v w \in E \\
\sum_{w \in G^{+}(v)} s^{\prime}(v w) & \geq a(v) N(v) & & \text { for all } v \in V \text { not a sink; } \\
\sum_{w \in G^{-}(v)} s^{\prime}(w v) & \leq b(v) N(v) & & \text { for all } v \in V
\end{aligned}
$$

For purposes of analysis, we will find it convenient to change notation slightly, at the same time allowing a slight generalisation. For each edge $v w \in E$, define

$$
s(v w)=s^{\prime}(v w) / b(w), \quad \alpha(v w)=b(w) / a(v) .
$$

The inequalities can now be written without reference to the functions $a(v)$ and $b(v)$, so we generalise to allow any positive function $\alpha(e)$ defined on edges. This gives us the following problem. 
Problem B. Suppose that $\alpha: E \rightarrow \mathbb{R}$ is a positive function. Maximise $N(Y) / N(X)$ subject to

$$
\begin{aligned}
N(v) & \geq 0 & & \text { for all } v \in V \\
s(v w) & \geq 0 & & \text { for all } v w \in E \\
\sum_{w \in G^{+}(v)} \alpha(v w) s(v w) & \geq N(v) & & \text { for all } v \in V \text { not a sink; } \\
\sum_{w \in G^{-}(v)} s(w v) & \leq N(v) & & \text { for all } v \in V .
\end{aligned}
$$

We clarify the exact meaning of Problem B by exposing the boundary cases. By a solution we will mean a pair of nonnegative functions $S=(N, s)$ satisfying (3). Define the value of a solution $S$ as

$$
f(S)= \begin{cases}-\infty & \text { if } N(X \cup Y)=0 \\ N(Y) / N(X) & \text { if } N(X)>0 \\ \infty & \text { if } N(X)=0, N(Y)>0 .\end{cases}
$$

Our task in Problem B is to find a solution $S=(N, s)$ that maximizes $f(S)$. Such a solution will be called optimal.

\section{A sample problem}

Consider a $k \times n$ array whose rows $\pi_{1}, \pi_{2}, \ldots, \pi_{k}$ are independent random permutations of $\{1,2, \ldots, n\}$. An intercalate is a 4 -tuple $\left(i_{1}, i_{2}, j_{1}, j_{2}\right)$ such that $1 \leq i_{1}<i_{2} \leq k, 1 \leq j_{1}<j_{2} \leq n, \pi_{i_{1}}\left(j_{1}\right)=\pi_{i_{2}}\left(j_{2}\right)$ and $\pi_{i_{1}}\left(j_{2}\right)=\pi_{i_{2}}\left(j_{1}\right)$. An intercalate is indicated in the following example for $n=9, k=4$.

\begin{tabular}{l|lllllllll}
3 & 7 & 1 & 2 & 8 & 9 & 4 & 5 & 6 \\
8 & 1 & 3 & 5 & 9 & 4 & 7 & 2 & 6 \\
6 & 9 & 5 & 8 & 3 & 7 & 1 & 4 & 2 \\
2 & 4 & 9 & 6 & 8 & 1 & 3 & 5 & 7
\end{tabular}

The number of intercalates has mean $\frac{1}{2}\left(\begin{array}{c}k \\ 2\end{array}\right)$ and can be almost as large as $\frac{n}{2}\left(\begin{array}{l}k \\ 2\end{array}\right)$ (the exact maximum is unknown). Our problem is to bound the probability that the number of intercalates is much larger than the mean.

Any particular intercalate can be destroyed by choosing one of its four entries and exchanging it with one of the $n-2$ entries in the same row that don't belong to the intercalate. This is the operation we will call a "switching". For example, exchanging the upper " 1 " in the above example with the " 6 " in the same row gives this:

$\begin{array}{lllllllll}3 & 7 & 1 & 2 & 8 & 9 & 4 & 5 & 6 \\ 8 & 6 & 3 & 5 & 9 & 4 & 7 & 2 & 1 \\ 6 & 9 & 5 & 8 & 3 & 7 & 1 & 4 & 2 \\ 2 & 4 & 9 & 6 & 8 & 1 & 3 & 5 & 7\end{array}$

Now we set up the optimization problem. The vertices of the graph are $\left\{v_{0}, v_{1}, \ldots\right\}$, where $v_{i}$ represents the set of arrays with $i$ intercalates. An edge from $v_{i}$ to $v_{j}$ means that a switching is possible from some array with $i$ intercalates to one with $j$ intercalates. A switching cannot destroy more than $2(k-1)$ intercalates at once, so $j-i \geq-2(k-1)$. A switching can create new intercalates as well, so some edges will have $j>i$. 
Given an array with $i$ intercalates, a switching can be done in $4 i(n-2)$ ways (choose an element of an intercalate and what to swap it with). So define $a\left(v_{i}\right)=$ $4 i(n-2)$. (Strictly speaking $a\left(v_{0}\right)$ is not supposed to be 0 , but since $v_{0}$ is a sink $a\left(v_{0}\right)$ does not appear in the constraints at all. We could set it to an arbitrary positive value, but we won't bother.) Conversely, given any array, we can use a reverse switching to create an intercalate by first choosing two rows and one column. This defines the two symbols that are involved unless they are the same. The other occurrences of those symbols in the two rows may already be in the same column (so an intercalate is there already), or can be brought into the same column by one of two possible swaps. So define $b\left(v_{i}\right)=2\left(\begin{array}{c}k \\ 2\end{array}\right) n$ for all $v_{i}$. (Note how $a\left(v_{i}\right)$ is a lower bound and $b\left(v_{i}\right)$ is an upper bound.) This is our instance of Problem A, or we can consider it an instance of Problem B with $\alpha\left(v_{i} v_{j}\right)=b\left(v_{j}\right) / a\left(v_{i}\right)=$ $k(k-1) n /(4 i(n-2))$.

This is all the problem-specific calculation we need. We will complete the solution in Section 6 after we develop the theory.

\section{Basic solutions}

We next describe some simple types of solution that we call basic.

By "path" we mean "simple directed path" and by "cycle" we mean "simple directed cycle". A loop is a cycle. If $F \subseteq E$ is a set of edges, then define $\alpha_{F}=$ $\prod_{e \in F} \alpha(e)$.

For convenience we define three pairs of functions, which are not necessarily solutions. If $P=\left(v_{0}, v_{1}, \ldots, v_{k}\right)$ is a path in $G$ from $v_{0}$ to $v_{k}(k \geq 0)$, then $S_{P}=\left(N_{P}, s_{P}\right)$ has $N_{P}=s_{P}=0$ except for $N\left(v_{k}\right)=1$ and in general

$$
\begin{aligned}
N_{P}\left(v_{i}\right) & =\alpha\left(v_{i} v_{i+1}\right) \cdots \alpha\left(v_{k-1} v_{k}\right) & & (0 \leq i \leq k) \\
s_{P}\left(v_{i-1} v_{i}\right) & =N_{P}\left(v_{i}\right) & & (1 \leq i \leq k) .
\end{aligned}
$$

$S_{P}$ satisfies (3) with equality everywhere, except that $(3 \mathrm{c})$ reads " $0 \geq 1$ " at $v_{k}$ and $(3 \mathrm{~d})$ reads " $0 \leq \alpha_{P}$ " at $v_{0}$.

If $C=\left(v=v_{0}, v_{1}, \ldots, v_{k}=v\right)$ is a cycle in $G(k \geq 1)$, then $S_{C, v}=\left(N_{C, v}, s_{C, v}\right)$ has $N_{C, v}, s_{C, v}=0$ except for

$$
\begin{aligned}
N_{C, v}(v) & =1 \\
N_{C, v}\left(v_{i}\right) & =s_{C, v}\left(v_{i-1} v_{i}\right)=\alpha\left(v_{i} v_{i+1}\right) \cdots \alpha\left(v_{k-1} v_{k}\right) \quad(1 \leq i \leq k-1) \\
s_{C, v}\left(v_{k-1} v\right) & =1
\end{aligned}
$$

$S_{C, v}$ satisfies (3) with equality everywhere, except that $(3 \mathrm{c})$ reads " $\alpha_{C} \geq 1$ " at $v$.

Under the same conditions, we also define the slightly different $\bar{S}_{C, v}=\left(\bar{N}_{C, v}, \bar{s}_{C, v}\right)$ which has $\bar{N}_{C, v}, \bar{s}_{C, v}=0$ except for

$$
\begin{aligned}
\bar{N}_{C, v}(v) & =\alpha_{C} \\
\bar{N}_{C, v}\left(v_{i}\right) & =\bar{s}_{C, v}\left(v_{i-1} v_{i}\right)=\alpha\left(v_{i} v_{i+1}\right) \cdots \alpha\left(v_{k-1} v_{k}\right) \quad(1 \leq i \leq k-1) \\
\bar{s}_{C, v}\left(v_{k-1} v\right) & =1
\end{aligned}
$$

$\bar{S}_{C, v}$ satisfies (3) with equality everywhere, except that $(3 \mathrm{~d})$ reads " $1 \leq \alpha_{C}$ " at $v$.

As the notation suggests, $S_{C, v}$ and $\bar{S}_{C, v}$ depend on which vertex $v$ is chosen as the starting vertex of the cycle.

Now we can define the basic solutions. Paths can have zero length and cycles can be loops, unless specified otherwise. 


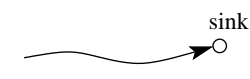

type 1

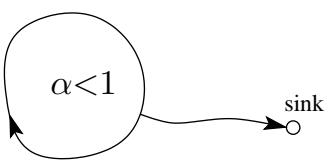

type 4

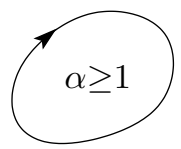

type 2

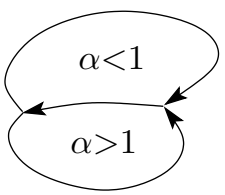

type 5

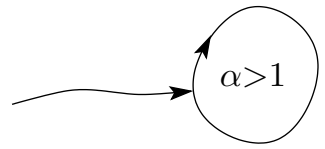

type 3

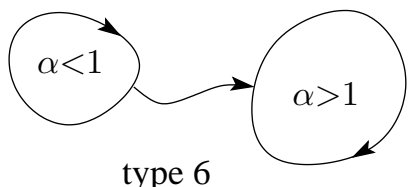

Figure 1. The supports of basic solutions

- Consider a path $P=\left(v_{0}, v_{1}, \ldots, v_{k}\right)$ with $v_{k}$ being a sink of $G$. A type-1 basic solution is any positive multiple of $S_{P}$.

- Consider a cycle $C=\left(v=v_{0}, v_{1}, \ldots, v_{k}=v\right)$ with $\alpha_{C} \geq 1$. A type-2 basic solution is any positive multiple of either $S_{C, v}$ or $\bar{S}_{C, v}$.

- Consider a path $P=\left(v_{0}, v_{1}, \ldots, v_{k}\right)$ of non-zero length, together with a cycle $C=\left(w=w_{0}, w_{1}, \ldots, w_{\ell}=w_{0}=w\right)$ with $\alpha_{C}>1$, such that $P$ and $C$ are disjoint apart from $v_{k}=w$. A type-3 basic solution is any positive multiple of $\left(\alpha_{C}-1\right) S_{P}+S_{C, w}$.

- Consider a path $P=\left(v_{0}, v_{1}, \ldots, v_{k}\right)$ of non-zero length, together with a cycle $C=\left(w=w_{0}, w_{1}, \ldots, w_{\ell}=w\right)$ with $\alpha_{C}<1$, such that $P$ and $C$ are disjoint apart from $v_{0}=w$. A type- 4 basic solution is any positive multiple of $\left(1-\alpha_{C}\right) S_{P}+\alpha_{P} \bar{S}_{C, w}$.

- Consider two cycles $C_{1}=\left(v=v_{0}, v_{1}, \ldots, v_{k}=v\right)$ and $C_{2}=(w=$ $\left.w_{0}, w_{1}, \ldots, w_{\ell}=w\right)$ which are disjoint apart from a common initial segment $v_{i}=w_{i}(0 \leq i \leq m)$ for some $0 \leq m<\min (k-1, \ell-1)$. Assume $\alpha_{C_{1}}<1$ and $\alpha_{C_{2}}>1$. A type-5 basic solution is any positive multiple of $\left(\alpha_{C_{2}}-1\right) S_{C_{1}, v}+\left(1-\alpha_{C_{1}}\right) S_{C_{2}, w}$.

- Consider two cycles $C_{1}=\left(v=v_{0}, v_{1}, \ldots, v_{k}=v\right)$ and $C_{2}=(w=$ $\left.w_{0}, w_{1}, \ldots, w_{\ell}=w\right)$, and a path $P=x_{0}, x_{1}, \ldots, x_{m}$ of non-zero length. These must be disjoint except that $v=x_{0}$ and $w=x_{m}$. Assume $\alpha_{C_{1}}<1$ and $\alpha_{C_{2}}>1$. A type-6 basic solution is any positive multiple of $\left(1-\alpha_{C_{1}}\right)\left(\alpha_{C_{2}}-1\right) S_{P}+\left(\alpha_{C_{2}}-1\right) \alpha_{P} \bar{S}_{C_{1}, v}+\left(1-\alpha_{C_{1}}\right) S_{C_{2}, w}$.

\section{Basic solutions suffice}

Theorem 1. Consider an instance $(G, \alpha, X, Y)$ of Problem B. Then if there are any non-zero solutions at all, there is an optimal solution which is basic.

Proof. The proof is divided into three cases, with almost the same method in each case.

(i) All solutions have $N(X)=N(Y)=0$.

(ii) Some solutions have $N(X)=0$ and $N(Y)>0$.

(iii) Some solutions have $N(X \cup Y)>0$ but for all such solutions $N(X)>0$. 
In each case we define a convex polytope for which some vertex must correspond to an optimal solution. Then we use the structure of the polytope to discover an optimal basic solution.

Consider case (i) first. Since positive multiples of solutions are also solutions, we can assume that $N(V)=1$. Adding this constraint to (3) defines a convex polytope $\mathcal{P}$ consisting of positive solutions. Suppose $S=(N, s)$ is a vertex of $\mathcal{P}$.

We will use the following principle. Suppose $T=\left(N_{T}, s_{T}\right)$ is a pair of functions, not identically 0 , such that $S+\varepsilon T$ satisfies (3) for all sufficiently small positive or negative $\varepsilon$. This implies that $N_{T}(X)=N_{T}(Y)=0$, by the definition of case (i). Also

$$
S+\varepsilon^{\prime}\left(T-N_{T}(V) S\right)=\left(1-\varepsilon^{\prime} N_{T}(V)\right)\left(S+\frac{\varepsilon^{\prime}}{1-\varepsilon^{\prime} N_{T}(V)} T\right)
$$

so $S+\varepsilon^{\prime}\left(T-N_{T}(V) S\right) \in \mathcal{P}$ for sufficiently small $\varepsilon^{\prime}$. Since $S$ is a vertex of $\mathcal{P}$, this is only possible if $T-N_{T}(V) S=0$, so $S$ is a multiple of $T$. We call such $T$ a free direction.

Let $H$ be the subgraph of $G$ induced by the edges $e$ with $s(e)>0$ and assume that $S$ is not a basic solution (implying, as above, that no free direction is a basic solution). We now consider a list of possibilities for $H$, in each case assuming that none of the earlier possibilities occur.

(a) Suppose $H$ has a cycle $C$ with $\alpha_{C}=1$. Let $v$ be a vertex of $C$ and define $T=S_{C, v}$, Then $T$ is a free direction since it satisfies (3) with equality. This can't happen, since $T$ is a type- 2 basic solution.

(b) Next suppose that $H$ has two different cycles $C_{1}, C_{2}$ with a common vertex $v$. Define $T=\left(N_{T}, s_{T}\right)=\left(\alpha_{C_{2}}-1\right) S_{C_{1}, v}+\left(1-\alpha_{C 1}\right) S_{C_{2}, v}$. Then $T$ satisfies (3) with equality, so $T$ is a free direction. But $S$ cannot be a multiple of $T$ for $\alpha_{C_{1}}, \alpha_{C_{2}}<1$ or $\alpha_{C_{1}}, \alpha_{C_{2}}>1$, since $s_{T}$ contains both positive and negative values. This also eliminates the case where the intersection of $C_{1}$ and $C_{2}$ is not a single segment, since then there is a third cycle $C_{3}$ having common vertices with each of $C_{1}$ and $C_{2}$, and it must be the case that either there are two of $\alpha_{C_{1}}, \alpha_{C_{2}}, \alpha_{C_{3}}$ less than 1 or two greater than 1 . The only case remaining is that $T$ is a type- 5 basic solution, which is false by assumption.

(c) Suppose $H$ has a cycle $C$ and a vertex $v$ of $C$ at which either (3c) or (3d) is a strict inequality. Then at least one of $S_{C, v}$ and $\bar{S}_{C, v}$ is a free direction. Both cases are impossible, since they violate (3) if $\alpha_{C}<1$ and are type-2 basic solutions if $\alpha_{C}>1$.

(d) Suppose $H$ has a (weak) component that is strongly-connected. Then the component must be a single vertex $v$, since all cases that include cycles have been eliminated. Then the type-1 basic solution $T=S_{(v)}$ (treating $v$ as a one-vertex path) is a free direction.

(e) Let $B$ be a source strong component of $H, B^{\prime}$ a sink strong component of $H$ lying in the same weak component, and $P$ a path from $B$ to $B^{\prime}$ which is disjoint from them except at its endpoints. Either $B$ is a single vertex or a cycle $C$ with $\alpha_{C}<1$ (the last following from (c)), and $B^{\prime}$ is either a single vertex (which must be a sink of $G$ by $(3 \mathrm{c}))$ or a cycle $C^{\prime}$ with $\alpha_{C^{\prime}}>1$. Let $T=\left(N_{T}, s_{T}\right)$ be the type-3, type- 4 or type- 6 basic solution that is based on $B \cup P \cup B^{\prime}$. In the case that $B$ is a single vertex $v$ with $N(v)=0$, modify $T$ by setting $N_{T}(v)=0$ also. Then $T$ is a free direction. The only case where $T$ is not a basic solution is the final modified 
one, but then a basic solution with the same value is obtained by further modifying $T$ by setting $s_{T}(e)=0$ for the edge $e$ of $P$ that leaves $v$.

That completes the proof for case (i). In case (ii), we define the polytope $\mathcal{P}$ using the constraint $N(Y)=1$ instead of $N(V)=1$. This is to ensure that the basic solution we derive has $N(Y)>0$. An optimal solution (with value $\infty$ ) is obtained by minimising the linear function $N(X)$, so we can assume the solution is at a vertex. The justification of "free direction" is the same except that in place of $T-N_{T}(V) S$ we use of $T-N_{T}(Y) S$, since in that direction the constraint $N(Y)=1$ is preserved.

For case (iii), we define $\mathcal{P}$ using the constraint $N(X)=1$. Having eliminated cases (i) and (ii), we know that the linear function $N(Y)$ is bounded. Otherwise, we would be able to find a solution with $N(Y)=1$ and $N(X)$ arbitrarily close to 0 , which is not possible unless we are in case (ii). The largest value of $N(Y)$ occurs at some vertex of $P$ and the rest of the argument is the same.

Theorem 1 allows us to immediately identify from the problem structure whether the optimal $f(S)$ of Problem B is finite or infinite. Define an elementary figure in $G$ to be one of the following subgraphs: a path ending at a sink, a cycle $C$ with $\alpha_{C} \geq 1$, and a path and a cycle $C$ with $\alpha_{C}>1$ such that the path and cycle are disjoint except that the last vertex of the path lies on the cycle.

Theorem 2. Consider an instance $(G, \alpha, X, Y)$ of Problem B. Then the following is true of an optimal solution $S$.

(a) If some elementary figure avoids $X$ but intersects $Y$, then $f(S)=\infty$.

(b) Otherwise, if some elementary figure intersects $X$, then $f(S)$ is finite.

(c) Otherwise, $f(S)=-\infty$.

Proof. Let the support of a solution $S=(N, s)$, be the subgraph $H=H(S)$ induced by the edges $e$ with $s(e)>0$. We know from Theorem 1 that (a)-(c) are true for the supports of basic solutions. These are elementary figures already for basic solutions of types 1, 2 and 3. For a type- 4 basic solution with support $H$, there is a type- 1 solution with support $H-w_{0} w_{1}$, which has the same vertex set. Similarly there is a type- 3 solution with the same vertex set as any type- 5 or type- 6 solution (remove edges $v_{m} v_{m+1}$ and $v_{0} v_{1}$, respectively).

In applications it can sometimes help to simplify the problem at the expense of a slight increase in the upper bound.

Lemma 1. Let $(G, \alpha, X, Y)$ be an instance of Problem B. Then none of the following operations can reduce the value of an optimal solution:

(a) increasing $\alpha(e)$ for an existing edge $e$;

(b) adding one edge vw with any value of $\alpha(v w)$, where $v$ is a vertex that already had at least one edge leaving;

(c) deleting all the edges leaving a particular vertex.

Proof. Let $S=(N, s)$ be an optimal solution of $(G, \alpha, X, Y)$. In each case, a solution $S^{\prime}=\left(N, s^{\prime}\right)$ for the new problem with $f\left(S^{\prime}\right)=f(S)$ is easily found. For (a), use $s^{\prime}=s$. For (b), use $s^{\prime}(v w)=0$ and $s^{\prime}(e)=s(e)$ for the existing edges. For (c), $s^{\prime}$ is the restriction of $s$ to the reduced edge set. 


\section{A special case}

In this section we analyse an important special case and find an upper bound on its solution that can be readily applied in many applications. Let $(G=(V, E), \alpha, X, Y)$ be an instance of Problem B. Define

$$
Z=\{v \in V: \alpha(v w) \geq 1 \text { for some } v w \in E, \text { or } v \text { is a sink of } G\} .
$$

We will make use of the following assumption.

$$
\text { A1. } Z \neq \emptyset, Y \cap Z=\emptyset, Z \subseteq X
$$

For $W, W^{\prime} \subseteq V$, define $\mathcal{Q}\left(W, W^{\prime}\right)$ to be the set of all non-trivial directed paths in $G$ that start in $W$, end in $W^{\prime}$, and have no internal vertices in $Y \cup Z$. Define

$$
\alpha_{Y Y}=\max _{Q \in \mathcal{Q}(Y, Y)} \alpha_{Q} \quad \text { and } \quad \alpha_{Y Z}=\max _{Q \in \mathcal{Q}(Y, Z)} \alpha_{Q},
$$

where the maximums over empty sets are taken to be 0 .

TheOREM 3. Let $S$ be an optimal solution of an instance of Problem B meeting Assumption A1. Then

$$
f(S) \leq \frac{\alpha_{Y Z}}{1-\alpha_{Y Y}}
$$

Proof. If $\mathcal{Q}(Y, Z)=\emptyset$ then $f(S) \leq 0$ by Theorem 2, so we will assume $\mathcal{Q}(Y, Z) \neq \emptyset$.

We begin by constructing a new graph $G^{\prime}=\left(V, E^{\prime}\right)$ from $G$ by, (a) deleting all the edges that leave vertices in $Z$ and, (b) for all $v, w \in Y$ with $v w \notin E$, add the edge $v w$ with $\alpha(v w)=\alpha_{Y Y}$. By Lemma 1 this can only increase the value of an optimal solution. Now consider the problem with $G^{\prime}$ replacing $G$. Since every edge $e$ of $G^{\prime}$ has $\alpha(e)<1$, Theorem 1 tells us that there is an optimal solution $S=(s, N)$ of type 1 or type 4 .

Let $H$ be the support of such an optimal solution. It consists of a path $P=$ $\left(v_{0}, v_{1}, \ldots, v_{k}\right)$ and possibly an extra edge $v_{m} v_{0}$ for some $0 \leq m<k$. If the extra edge is present in $H$, let $C$ be the cycle so formed; otherwise put $C=\emptyset$. Our assumptions and definition of $G^{\prime}$ imply that $P \cap Z=\left\{v_{k}\right\}$. Normalise $S$ so that $N\left(v_{k}\right)=1$. Then for $0 \leq i \leq k$ we have

$$
N\left(v_{i}\right)= \begin{cases}\alpha\left(v_{i} v_{i+1}\right) \cdots \alpha\left(v_{k-1} v_{k}\right) & \text { if } v_{i} \notin C ; \\ \frac{\alpha\left(v_{i} v_{i+1}\right) \cdots \alpha\left(v_{k-1} v_{k}\right)}{1-\alpha} & \text { if } v_{i} \in C .\end{cases}
$$

Since $v_{k} \in X$ and $N\left(v_{k}\right)=1, f(S) \leq N(Y)$. Since the theorem will come from this upper bound, we will assume that $H$ is the support of the type- 1 or type- 4 basic solution which maximizes $N(Y)$ (even if it isn't an optimal solution).

We now consider three operations on $H$ that cannot decrease $N(Y)$ for the type-1 or type- 4 basic solution with support $H$.

- Suppose the solution is type-1 $(C=\emptyset)$ and $v_{0} \notin Y$. Then removing $v_{0}$ from $H$ leaves $N(Y)$ unchanged.

- Suppose the solution is type-4 and $C \cap Y=\emptyset$. Then removing $C$ from $H$ leaves $N(Y)$ unchanged.

- Suppose $H$ has a subpath or cycle $H^{\prime}=\left(w_{0}, w_{1}, \ldots, w_{\ell}\right)$, where $w_{0}=w_{\ell}$ if $H^{\prime}$ is a cycle. Suppose $\ell \geq 2$ and $H^{\prime} \cap Y=\left\{w_{0}, w_{\ell}\right\}$. 
- Suppose $C=\emptyset$ or $v_{m} \notin\left\{w_{1}, \ldots, w_{\ell-1}\right\}$. Then delete $\left\{w_{1}, \ldots, w_{\ell-1}\right\}$ from $H$ and insert the edge $w_{0} w_{\ell}$ into $H$. By the definition of $\alpha_{Y Y}$, this operation cannot decrease $N(Y)$.

- Suppose instead that $C \neq \emptyset$ and $v_{m}=w_{j} \in\left\{w_{1}, \ldots, w_{\ell-1}\right\}$. Then delete $\left\{w_{j+1}, \ldots, w_{\ell-1}\right\}$ from $H$ and insert the edge $w_{0} w_{\ell}$ into $H$. Again, by the definition of $\alpha_{Y Y}$, this operation cannot decrease $N(Y)$.

It follows from these considerations that we may assume $P \cap Y=\left\{v_{0}, v_{1}, \ldots, v_{t}\right\}$, where $0 \leq t<k$ for a type-1 solution and $m \leq t<k$ for a type-4 solution. So $H \cap Y$ is connected and $C \subseteq Y$. In the case of a type- 1 solution, we have

$$
N(Y) \leq N\left(v_{t}\right)\left(1+\alpha_{Y Y}+\cdots+\alpha_{Y Y}^{t}\right)<\frac{N\left(v_{t}\right)}{1-\alpha_{Y Y}} .
$$

In the case of a type- 4 solution, we have

$$
N(Y) \leq N\left(v_{t}\right)\left(1+\alpha_{Y Y}+\cdots+\alpha_{Y Y}^{t-m-1}+\frac{\alpha_{Y Y}^{t-m}+\cdots+\alpha_{Y Y}^{t}}{1-\alpha_{Y Y}^{m+1}}\right)=\frac{N\left(v_{t}\right)}{1-\alpha_{Y Y}} .
$$

Since any edges of $G^{\prime}$ not present in $G$ are within $Y, N\left(v_{t}\right) \leq \alpha_{Y Z}$ and the theorem follows.

To apply Theorem 3 we need upper bounds on $\alpha_{Y Y}$ and $\alpha_{Y Z}$. This may not be simple, but there are some fairly general cases. We consider three possible assumptions, each of which comes with a definition of a quantity $\bar{\alpha}$.

A2. Suppose there is a positive function $\beta: V \times V \rightarrow \mathbb{R}$ such that $\alpha(v w)=$ $\beta(v, w)$ for each $v w \in E$. Moreover, for all distinct $v, w, x$ with $w \notin Y \cup Z$ we have $\beta(v, w) \beta(w, x) \leq \beta(v, x)$. Define $\bar{\alpha}=\max _{v, w \in Y} \beta(v, w)$.

$\mathrm{A} 2^{\prime}$. In the case of Problem A, $b(v) \leq a(v)$ for $v \notin Y \cup Z$. Define $\bar{\alpha}=$ $\max _{v, w \in Y}(b(v) / a(w))$.

$\mathrm{A} 2^{\prime \prime}$. Suppose there is a positive function $p: V \rightarrow \mathbb{R}$ such that $\alpha(v w)=p(v)$ for all $v w \in E$. Define $\bar{\alpha}=\max _{v \in Y} p(v)$.

A path $\left(v_{0}, v_{1}, \ldots, v_{k}\right)$ is shortcut-free if there is no edge $v_{i} v_{j}$ for $0 \leq i, j-i \geq$ $2, j \leq k$.

Lemma 2. Let $S$ be an instance of Problem $B$ meeting Assumption $A 1$ and one of $A 2, A 2^{\prime}, A 2^{\prime \prime}$. Then $\alpha_{Y Y} \leq \bar{\alpha}$. Furthermore, if $\mathcal{Q}(Y, Z) \neq \emptyset$, then $\alpha_{Y Z}$ is realised by some shortcut-free path.

Proof. If $Q=\left(v_{0}, \ldots, v_{k}\right) \in \mathcal{Q}(Y, Y)$, then Assumption A2 implies that $\alpha_{Q} \leq$ $\beta\left(v_{0}, v_{k}\right)$. Similarly $\alpha_{Q}$ for $Q \in \mathcal{Q}(Y, Z)$ cannot be reduced by taking a shortcut. Assumptions $\mathrm{A} 2^{\prime}$ and $\mathrm{A} 2^{\prime \prime}$ are seen to be special cases of $\mathrm{A} 2$ : define $\beta(v, w)=$ $b(w) / a(v)$ and $\beta(v, w)=p(v)$, respectively.

In many common cases, $V$ has a natural ordering according to some parameter and the edges of $G$ are limited in how much they can decrease the parameter. We formalise this in another assumption.

A3. $V=\left\{x_{0}, x_{1}, \ldots, x_{n}\right\}$ for some $n$. Furthermore, there are integers $M>N$ and $K>0$ such that $Y=\left\{x_{i}: i \geq M\right\}, Z=\left\{x_{i}: i \leq N\right\}$ and for every edge $x_{i} x_{j}$ we have $j-i \geq-K$.

In the following we use "increasing" and "decreasing" in their non-strict senses. 
Lemma 3. Let $S$ be an instance of Problem $B$ meeting Assumptions A1, A3

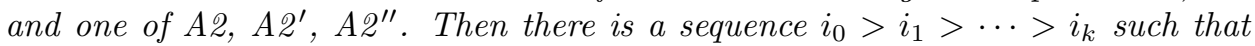
$i_{0} \geq M, i_{1}<M, i_{k-1}>N, i_{k} \leq N, i_{j}-i_{j+1} \leq K$ for $j=0, \ldots, k-1$, and

$$
\alpha_{Y Z} \leq \begin{cases}\prod_{j=1}^{k} \beta\left(x_{i_{j-1}}, x_{i_{j}}\right) & \text { for } A \mathscr{2}, \\ \prod_{j=1}^{k} b\left(x_{i_{j}}\right) / \prod_{j=0}^{k-1} a\left(x_{i_{j}}\right) & \text { for } A \mathscr{2}^{\prime}, \\ \prod_{j=0}^{k-1} p\left(x_{i_{j}}\right) & \text { for } A \mathscr{2}^{\prime \prime} .\end{cases}
$$

Furthermore, under any of the following assumptions, it can be assumed that $i_{j}=$ $M-j K$ for $j=0, \ldots, k-2$ and $i_{k-1}=\max \{M-(k-1) K, N+1\}$.

(a) for A2, $\beta\left(x_{i}, x_{j}\right)$ is a decreasing function of $i$ and $j$;

(b) for $A 2^{\prime}, a\left(x_{i}\right)$ is an increasing function of $i$, and $b\left(x_{i}\right)$ is a decreasing function of $i$;

(c) for $A 2^{\prime \prime}, p(i)$ is a decreasing function of $i$. In this case we can also assume $i_{k-1}=M-(k-1) K$.

Proof. To take the worst case, we can assume that all edges $x_{i} x_{j}$ with $j-$ $i \geq-K$ are present. With this assumption, let $Q=\left(x_{i_{0}}, \ldots, x_{i_{k}}\right) \in \mathcal{Q}(Y, Z)$ maximize $\alpha_{Q}$, and subject to that be lexicographically minimal. The lexicographic minimality shows that $Q$ is shortcut-free (and therefore $i_{0}>i_{1}>\cdots>i_{k}$ ). It further shows that $i_{j}=M-j K$ for $j=0, \ldots, k-2$, since otherwise we can reduce some $i_{j}$ by one to violate the minimality without reducing $\alpha_{Q}$. In the case of (c), if $i_{k-1}=N+1>i_{k-2}-K$, then $\alpha_{Q}$ is not decreased if we decrease $i_{k-1}$ by one and delete $i_{k}$.

A numerical lemma which can be useful for turning Lemma 3 into an explicit formula is as follows.

LEMMA $4([4])$. Let $k$ be a positive integer and let $q$ and $s$ be positive real numbers such that $s \geq k q$. Then

$$
s(s-q) \cdots(s-(k-1) q) \geq s^{k} \exp \left(-k^{2} q / s\right) \geq(s / e)^{k} .
$$

For convenience we give a moderately sharp bound for a common case.

Corollary 1. Suppose $V=\left\{x_{0}, x_{1}, \ldots, x_{n}\right\}$ where $x_{0}$ is the only sink, and that $Y=\left\{x_{i}: i \geq M\right\}$. Also suppose that for some $K>0$, each edge $x_{i} x_{j}$ has $j-i \geq-K$ and $\alpha\left(x_{i} x_{j}\right)=\rho / i$, where $\rho>0$ is constant. Then for integer $M>\max \{\rho, K-1\}$,

$$
f(S) \leq \frac{1}{1-\rho / M}(\rho / M)^{k} \exp \left(k^{2} K / M\right) \leq \frac{1}{1-\rho / M}(e \rho / M)^{k},
$$

where $k=\lfloor(M+\min \{0, K-\rho-1\}) / K\rfloor$.

Proof. This case matches Lemma 3(c) with $N=\lfloor\rho\rfloor$. Also $\bar{\alpha} \leq \rho / M$. Now apply Theorem 3 and Lemma 4 . The given value of $k$ satisfies the requirements that $k \leq M / K$ needed for Lemma 4 and $M-(k-1) K \geq N$ needed for Lemma $3(\mathrm{c})$.

\section{Sample applications}

We first complete the sample problem we started in Section 2. Then we give two more examples. 


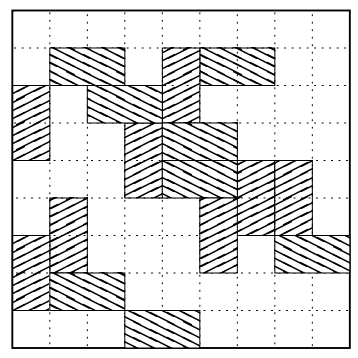

Figure 2 . A $9 \times 9$ board with 16 dominoes covering $62 \times 2$ subsquares

For the problem of Section 2, we can apply Corollary 1 . For $n \geq 8$, we can use $\rho=\frac{1}{3} k(k-1)$ and $K=2(k-1)$. For example, for $\lambda \geq 2$ the probability that there are more than $M=\lambda\left(\begin{array}{l}k \\ 2\end{array}\right)$ intercalates ( $M$ integer) is at most

$$
2\left(\frac{2 e}{3 \lambda}\right)^{\lambda k / 4-k / 6-1}
$$

Cliques in random graphs. Given a random graph with $n$ vertices and $m$ edges, what is the probability that the number of 4-cliques is large?

A switching consists of choosing a 4-clique, deleting its 6 edges, and inserting them anywhere in the graph (even if it creates the same 4-clique). If there are $i$ 4-cliques, this can be done in at least

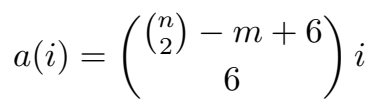

ways. The reverse operation is to choose 6 edges and make a 4-clique from them, which can be done in at most

$$
b(i)=\left(\begin{array}{c}
m \\
6
\end{array}\right)\left(\begin{array}{l}
n \\
4
\end{array}\right)
$$

ways. A switching cannot destroy more than $6 m$ 4-cliques at once. Therefore, we can apply Corollary 1 with $K=6 \mathrm{~m}$.

Dominoes on a chessboard. Consider an $n \times n$ chessboard and $d$ dominoes. Place the dominoes at random on the board in non-overlapping positions, with all possible configurations being equally likely. What is the probability that the number of $2 \times 2$ squares covered by dominoes is large?

A switching consists of choosing a covered $2 \times 2$ square, removing the domino covering its upper left quadrant, and putting the domino down somewhere (even in the same place). If there are $i$ covered $2 \times 2$ squares, this can be done in at least

$$
a(i)=(2 n(n-1)-7 d) i
$$

ways, since one domino can eliminate up to 7 domino positions. Conversely, choose any domino and move it to a place where it covers the upper left quadrant of a $2 \times 2$ subsquare which is now covered. This can be done in at most

$$
b(i)=2 d^{2}
$$

ways since there can't be more than $2 d$ such ways to place one domino and there were $d$ dominoes to choose from. One switching can eliminate up to 6 covered subsquares. Therefore, we can apply Corollary 1 with $K=6$. 


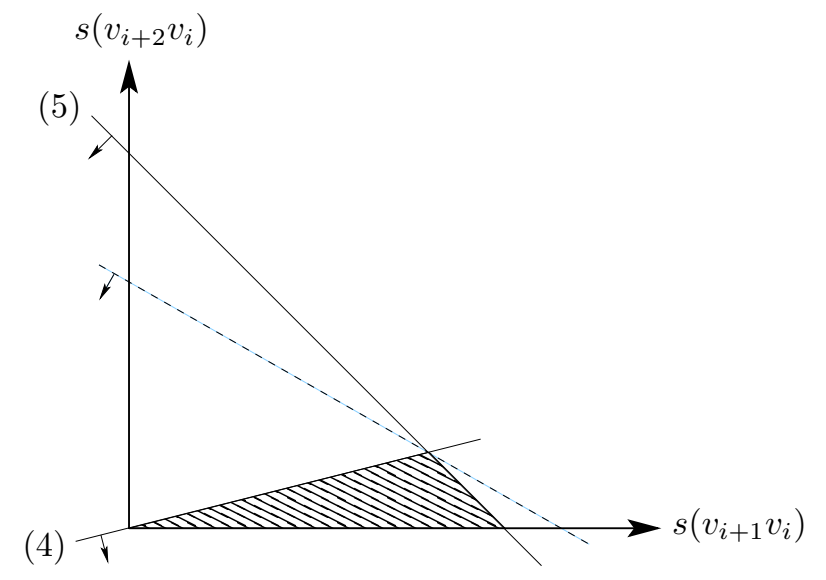

FiguRE 3. Approximating an extra constraint

Extra constraints. This example is artificially simplified in order to illustrate an extra technique. Suppose we have a family of objects with non-negative integer weights. Let $C\left(v_{i}\right)$ denote the set of all objects of weight $i$, and let $N\left(v_{i}\right)=\left|C\left(v_{i}\right)\right|$. We also have a switching operation which takes each object of weight $i$ onto at least two objects of weight $i-1$ or $i-2$. Moreover, a reverse switching takes each object of weight $i$ onto at most one object of weight $i+1$ or $i+2$.

Now we seek an upper bound on $N\left(v_{n}\right) / N\left(v_{0}\right)$, where we assume $n$ is even for simplicity. This is an elementary case of Problem A, with $a\left(v_{i}\right)=2$ and $b\left(v_{i}\right)=1$ for each $i$. Converting to Problem B as in (2), we find that each edge $e$ has $\alpha(e)=\frac{1}{2}$. Only type- 1 solutions are possible, so the solution in Lemma 3 is best possible. We find that an optimal path is $\left(v_{n}, v_{n-2}, \ldots, v_{2}, v_{0}\right)$, and therefore $N\left(v_{n}\right) / N\left(v_{0}\right) \leq$ $2^{-n / 2}$.

Now suppose we have the following additional information: on average for the objects of weight $i$, at least a fraction $\frac{99}{100}$ of reverse switchings produce an object of weight $i-1$ and at most $\frac{1}{100}$ produce an object of weight $i-2$. In the language of Problem A, this corresponds to a constraint $s^{\prime}\left(v_{i+2} v_{i}\right) \leq \frac{1}{99} s^{\prime}\left(v_{i+1} v_{i}\right)$, for each $i \leq n-2$, which after conversion to Problem B becomes

$$
s\left(v_{i+2} v_{i}\right) \leq \frac{1}{99} s\left(v_{i+1} v_{i}\right) .
$$

In general, our precise analysis does not cover such constraints, and Theorem 1 does not apply, but we can work with an approximation. Constraint (3d) gives

$$
s\left(v_{i+1} v_{i}\right)+s\left(v_{i+2} v_{i}\right) \leq N\left(v_{i}\right) .
$$

Constraints (4) and (5) define a region like the shaded triangle in Figure 3. We will replace them by a single constraint like that shown in the figure by a dashed line.

The general form of the new constraint is

$$
\bar{s}\left(v_{i+1} v_{i}\right)+\bar{s}\left(v_{i+2} v_{i}\right) \leq N\left(v_{i}\right)
$$

where

$$
\bar{s}\left(v_{i+1} v_{i}\right)=\frac{100}{99+\Delta} s\left(v_{i+1} v_{i}\right) \text { and } \bar{s}\left(v_{i+2} v_{i}\right)=\frac{100 \Delta}{99+\Delta} s\left(v_{i+2} v_{i}\right)
$$


for some $\Delta \geq 1$. If we now define

$$
\bar{\alpha}\left(v_{i+1} v_{i}\right)=\frac{99+\Delta}{200} \text { and } \bar{\alpha}\left(v_{i+2} v_{i}\right)=\frac{99+\Delta}{200 \Delta},
$$

for each $i$, then constraint (3c) becomes

$$
\bar{\alpha}\left(v_{i} v_{i-1}\right) \bar{s}\left(v_{i} v_{i-1}\right)+\bar{\alpha}\left(v_{i} v_{i-2}\right) \bar{s}\left(v_{i} v_{i-2}\right) \geq N\left(v_{i}\right) .
$$

Constraints (6) and (7) define a new instance of Problem B. We now choose $\Delta$ to optimise the solution. A reasonable value is $\Delta=\frac{99}{50}$, which gives

$$
\bar{\alpha}\left(v_{i} v_{i-1}\right) \bar{\alpha}\left(v_{i-1} v_{i-2}\right)<\bar{\alpha}\left(v_{i} v_{i-2}\right)=\frac{51}{200} .
$$

The optimal path is thus $\left(v_{n}, v_{n-2}, \ldots, v_{2}, v_{0}\right)$ again, and we obtain the considerably better bound $N\left(v_{n}\right) / N\left(v_{0}\right) \leq\left(\frac{51}{200}\right)^{n / 2}$.

This technique can be generalized considerably, but the details remain to be worked out.

\section{Conclusions}

We have demonstrated a new technique for bounding the tails of distributions and other similar tasks. An open problem is to extend the theory a probabilistic setting, where the quantities $N(v)$ are random variables and the quantities $s(e)$ are expectations.

\section{References}

[1] C. Cooper, A. Frieze, B. Reed and O. Riordan, Random regular graphs of non-constant degree: independence and chromatic number, Combin. Probab. Comput., 11 (2002) 323-341.

[2] V. Fack and B.D. McKay, A generalized switching method for combinatorial estimation, Australasian J. Combin., 39 (2007) 141-154.

[3] C. D. Godsil and B.D. McKay, Asymptotic enumeration of Latin rectangles, J. Combin. Theory Ser. B, 48 (1990) 19-44.

[4] C. S. Greenhill and B. D. McKay, Asymptotic enumeration of sparse nonnegative integer matrices with specified row and column sums, Adv. Appl. Math., 41 (2008) 459-481.

[5] S. Janson and A. Ruciński, The infamous upper tail, Random Structures Algorithms, 30 (2002) 317-342.

[6] M. Krivelevich, B. Sudakov, V.H. Vu and N. C. Wormald, Random regular graphs of high degree, Random Structures Algorithms, 18 (2001) 346-362.

[7] P. Lieby, B. D. McKay, J. C. McLeod and I. M. Wanless, Subgraphs of random $k$-edge-coloured k-regular graphs, Combin. Probab. Comput., 18 (2009) 533-549.

[8] B. D. McKay, Subgraphs of random graphs with specified degrees, Congr. Numer., 33 (1981) $213-223$.

[9] B. D. McKay, Asymptotics for 0-1 matrices with prescribed line sums, in Enumeration and Design, (Academic Press, 1984) 225-238.

[10] B. D. McKay and I. Wanless, Most Latin squares have many subsquares, J. Combin. Theory Ser A, 86 (1999) 323-347.

[11] B. D. McKay, N. C. Wormald and B. Wysocka, Short cycles in random regular graphs, Electron. J. Combin., 11 (2004), \#R66, 12 pages.

Department of Computer Science, Faculty of Mathematics and Computer Science Amirkabir University of Technology, Tehran, Iran

E-mail address: m.hashemi@aut.ac.ir

Department of Computer Science, Australian national University, Canberra ACt 0200, Australia

E-mail address: bdm@cs.anu.edu.au 